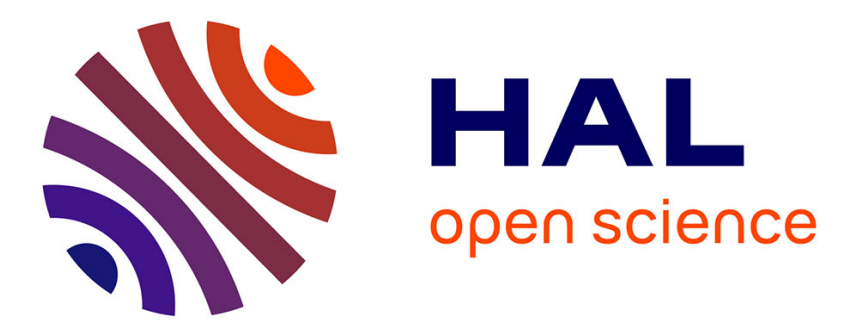

\title{
Solid-fluid transition modelling in geomaterials and application to a mudflow interacting with an obstacle
}

Noemie Prime, Frédéric Dufour, Félix Darve

\section{To cite this version:}

Noemie Prime, Frédéric Dufour, Félix Darve. Solid-fluid transition modelling in geomaterials and application to a mudflow interacting with an obstacle. International Journal for Numerical and Analytical Methods in Geomechanics, 2014, 38 (13), pp.1341 - 1361. 10.1002/nag.2260 . hal-01815564

\section{HAL Id: hal-01815564 \\ https://hal.univ-smb.fr/hal-01815564}

Submitted on 14 Jun 2018

HAL is a multi-disciplinary open access archive for the deposit and dissemination of scientific research documents, whether they are published or not. The documents may come from teaching and research institutions in France or abroad, or from public or private research centers.
L'archive ouverte pluridisciplinaire HAL, est destinée au dépôt et à la diffusion de documents scientifiques de niveau recherche, publiés ou non, émanant des établissements d'enseignement et de recherche français ou étrangers, des laboratoires publics ou privés. 


\title{
Solid-fluid transition modelling in geomaterials and application to a mudflow interacting with an obstacle
}

\author{
Noémie Prime ${ }^{1}$, Frédéric Dufour ${ }^{2}$, Félix Darve ${ }^{2} 3$
}

Given the contrasting behaviour observed for geomaterials, for example during landslides of the flow type, this contribution proposes an original constitutive model which associates both an elasto-plastic relation and a Bingham viscous law linked by a mechanical transition criterion. This last is defined as the second-order work sign for each material point, which is a general criterion for divergence instabilities. FEMLIP method (Finite Element Method with Lagrangian Integration Points) is chosen as a framework for implementing the new model because of its well-known ability to deal with both solid and fluid behaviours in large deformation processes. A first boundary model considering a sample of initially stable soil, a slope, and an obstacle is performed. The results show the power of the constitutive model since the consistent evolution of initiation, propagation and arrest of the mudflow is described. A parametric study is led on both plastic and viscous parameters to determine their influence on the flow development and arrest. Finally, forces against the obstacle are compared with good agreement with those of other authors for the same geometry and a pure viscous behaviour.

keywords : failure, landslides, second-order work, solid-fluid transition, elasto-plasticity, non-newtonian viscosity.

\section{Introduction}

\subsection{Behaviour transition in different granular media}

Understanding and well describing solid-fluid transitions in geomaterials, or more generally in any granular material, represents a scientific key-point, and many works are being done in this direction, by the physicists for the dry state $[1,2]$ as well as by the rheologists for the saturated state $[3,4]$. The very particular behaviour of a granular medium plays a major role in these transitions. For instance, it has been admitted that a dry granular medium presents -in quasi-static conditions- a bimodal behaviour of the macroscopic variables $[5,6]$, and thus could be usually separated in two distinct phases, one with 'strong' contact network, which can support deviatoric stresses without being strained, and one with 'weak' contact network, in which the deviatoric stresses are dissipated due to the shear strains. Moreover, for an inclined layer of grains, if the slope angle is ranged from the repose angle (maximum angle for an unconditional stability of the granular structure) to the avalanche angle (minimum angle for an unconditional instability), the system can respond by one state or another with an unstable behaviour [7]. Figure 1 shows, for a granular media subjected to the rotation of a drum, a

\footnotetext{
${ }^{1}$ Université de Liège, Chemin des Chevreuils 1, 4000 Liège, Belgique, noemie.prime@ulg.ac.be

${ }^{2}$ Univ. Grenoble Alpes, 3SR, F-38041 Grenoble

${ }^{3}$ CNRS, 3SR, F-38041 Grenoble
} 
flowing layer developed at the surface. The two phases appear well separated here. In addition to that observation, it can be mentioned that some constitutive models consider a dependency of the behaviour on a free energy function which admits 2 minima : one for fully mobile grains and one for static grains [8]. This could mean that the static/mobile transition is instantaneous without stable configuration between the two states.

Saturated granular media also undergo such a transition, as -for non zero slopes- granular suspensions can flow or freeze. The going through between the two states has been observed to be very sudden, with a velocity that instantaneously pass -for an infinitesimal increase of shear stress- from zero to a significant value (see some results of Huang [9]).

In the context of natural hazards, some kinds of landslides, as mudflows or debris flows, are also characterized by an extremely rapid motion of the soil (several meters/second) under a fluidized form. For instance, landslides in Gansu (China, august 7th 2010), or in Sarno and Quindici (Italy, may 5th 1998) have developed in such a way. In those events, the finest part of the bulk mass forms a granular suspension since it is a mixture of water and particles whose dimension is globally greater than $1 \mathrm{~nm}$. The geomaterial thus reached a specific state where the behaviour changes from a solid type (for the in situ soil) to a complex-fluid type (for the suspension).

In order to mitigate the risks, the natural question is: how to describe -in a unique numerical framework- the soil solid-fluid transition leading to the mud or debris flow, that is to say both the initiation and the propagation of the flow, and even its final arrest? This question is moreover of high interest since landslides of the flow type are particularly dangerous due to their high velocity and their long run-out. For instance, Gansu mudslide killed more than 1470 persons and Sarno and Quindici one caused 161 fatalities. Numerical predictive models represent thus a strong societal issue.

\subsection{Actual limitations for modelling solid-fluid transitions}

The first limitation concerns the constitutive model to describe the behaviour of a solid soil which evolves toward a real fluid state. To the author's knowledge, not any unified relation has been written for that.

In fluid rheology, some models have been proposed to deal with such a transition with a pure viscous relation, in which the viscosity drastically varies, at a certain stress threshold, between low, physical, values up to values tending to infinity, which is a numerical way to induce a quasi-solidification of the material. But the drawback is that no real solid behaviour is described as the whole constitutive relation is viscous. Coussot et al. [10] defined such a constitutive relation to reproduce the flowing and the jamming of bentonite suspensions, in considering, moreover, the history (the thixotropy) of the behaviour. Other constitutive relations define a jamming stress criterion beyond which viscous strains are activated. Many models belong to this family: from very simple ones (for instance Bingham's model - figure 4.a) to much elaborated ones (for example the model developed by Jop et al. [11] for dense granular flows). However, their main drawback is that, even if the flowing domain is bounded by what can be considered as a solid-fluid transition criterion, the solid behaviour inside this surface (in the stress space) is not defined.

In solid mechanics, and in particular in geomechanics, carrying on describing the material behaviour after the failure is a particularly difficult issue. Besides, viscosity is considered in some visco-elasto-plastic models (models of Perzyna's type), but mainly to describe the plastic flow. In perfect plasticity, these models describe the development of viscous strain rates only 
once the stress state oversteps the plastic limit criterion. However, it has been shown that a disorganisation of the soil and a fluidized evolution of it could happen without verifying this criterion (see section 2). Besides, when hardening is considered, Perzyna's models describe a progressive evolution from a plastic flow to a viscous one, but not a sudden solid-fluid transition as it can be observed in granular media (see previous paragraph), in granular suspension [9], and for certain types of failure in in-situ soils (see section 2). To conclude, none of the existing constitutive models are not entirely satisfying concerning the soils solid-fluid transition description.

The second limitation concerns the numerical method which must be able to describe -in a unique model- both solid and liquid phases which may moreover coexist in the domain of interest.

First, although discrete element methods are very flexible and suited -at a small scale- to describe some complex responses of granular media (in a dry state or not), it would take too much computational time to use them at the large scale of natural flows. Besides, studying mud or debris flows with continuum methods is really challenging because they are usually specific for the modelling of either fluids or solids. For instance, on one hand, Eulerian Finite Element Method (FEM) can account for the very high level of deformation of fluids (the mesh is kept fixed such as no element distortion prevent the computation from converging). However, the Eulerian advection procedure induces numerical diffusion of material variables and decreases the computational accuracy for history-dependent materials. On the other hand, a Lagrangian formulation of FEM, for which the mesh is always connected with the material, allows a very accurate tracking of internal variables, which is necessary to model solid behaviours and notably to take into account the history parameters. However, in this case the computational accuracy is limited by the mesh tangling due to the level of displacements. A great deal of effort is nowadays done to make numerical methods more versatile, as for example the Smooth Particles Hydrodynamics method (SPH) [12, 13], initially applied in geomechanics for fluid geomaterials and recently adapted also for solid elasto-plastic geomaterials [14].

Considering these actual limitations in the initiation and propagation modelling of landslides of the flow type, two original advances are proposed in this contribution:

(i) The development of a new constitutive model, able to describe the elasto-plastic behaviour of an in situ soil, to detect -with an accurate criterion- if solid-fluid transition occurs and to describe the viscous behaviour of the flowing geomaterial.

(ii) An application of this transition model on a simple boundary value problem by means of a powerful numerical method, called Finite Element Method with Lagrangian Integration Point (FEMLIP), suitable for both fluid and solid behaviours.

For this first application, inertial effects are disregarded, and hydromechanical coupling are considered only through a modification of cohesion.**

This paper is organized as follows. In section 2, the unified constitutive model elaborated to describe the solid-fluid transition is presented, keeping focused on the failures of the diffuse type, and clarifying the link between the failure and the transition state. In section 3, the bases of the FEMLIP numerical method are recalled, and its advantages in comparison with the classical methods are highlighted. Section 4 presents the simplified case chosen for a first simulation, using the new transition model implemented in a FEMLIP code. Various cases 
are considered, notably a study of the influence of a plastic parameter and a viscous one. In section 5, a comparison is proposed between our results and those, for the same geometrical model, of other authors. Finally, section 6 draws some conclusions about the efficiency of the new constitutive model used with FEMLIP method and some possible prospects.

\section{A new constitutive model for diffuse solid-fluid tran- sition in geomaterials}

\subsection{Reaching the failure state in geomaterials}

\subsubsection{Diversity of failure phenomena}

Landslides are obviously linked to instabilities in soils, which means that at a given state of the material, an infinitesimal load induces a large response.

Globally, instabilities can be due whether to specific boundary conditions (they are called 'geometric instabilities') or to the properties and state of the material itself (they are called 'material instabilities'). Instabilities in geomaterials, which generally concern a bulk of material without any specific geometry, are most of the time of the 'material' type. Moreover, 'material instabilities' gather instabilities for which the strains increase suddenly at a certain stage of the loading (they are named 'divergence instabilities') and instabilities for which strains evolve cyclically with an increasing amplitude, the so-called 'flutter instabilities' [15]. In geomechanical context, instabilities are mainly of the divergence type.

Different modes of failure can anyway be distinguished among divergence instabilities. At least, 2 modes are classically highlighted : localized and diffuse modes. Localisation leads to a concentration of the plastic strains in a shear band and has been well investigated. On the other hand, diffuse failure appears without any local concentration of strains, but with a response in the bulk which corresponds to a global disorganisation of the soil structure [16]. In such a case, the nature of the grain contact is evolving, and the inter-granular stresses are decreasing. This collapse can be observed for an undrained triaxial test on a loose sand (figure 3). In this particular non-cohesive material, the contacts between grains completely vanish and the final stress tensor ends equal to zero. It thus corresponds to liquefaction.

\subsubsection{Limitation of the classical failure criterion in case of diffuse failures}

An analysis of diffuse failure has only been recently achieved [17, 18, 19], notably because in this case the failure stress state has the specificity to not correspond with the plastic limit criterion.

Thus, to predict such kind of failure mode, nor limit plasticity theory neither localization theory [20] are suitable $[18,21]$. This can be express as follows:

(i) the plastic limit condition $(\operatorname{det}(\boldsymbol{M})=0$, with $\boldsymbol{M}$ being the elasto-plastic constitutive matrix) is not necessarily fulfilled at failure. Let us recall that matrix $\boldsymbol{M}$ links the strain and stress increments (written in engineering notations) as follows : $\boldsymbol{d} \boldsymbol{\sigma}=\boldsymbol{M} \boldsymbol{. d} \boldsymbol{\varepsilon}$.

(ii) the localization criterion $(\operatorname{det}(\boldsymbol{L})=0$, with $\boldsymbol{L}$ being the acoustic tensor) is not either always met at failure.

This is due to the non-associativeness property of soils -contrary to associated materials as metals [22]- which implies the non-symmetry of the constitutive matrix $\boldsymbol{M}$. 
For instance, landslides as the one in Petacciato (Italy) have developed with a very low slope, around $6^{\circ}$, compared to the friction angle of the involved material, around $19^{\circ}$ [23]. Thus, limit plasticity theory as well as localization theory are not sufficient to determine the stability status, which is a strong limitation regarding the safety of geotechnical works or landslides prediction.

\subsection{The second-order work transition criterion}

Hill [24] has developed a theory of the general stability for solids. In the hypothesis of small strains and negligible geometrical effects, the sufficient stability condition is expressed, for a material point, as follows:

$$
d^{2} W=d \sigma_{i j} d \varepsilon_{i j}>0, \quad \forall\|\boldsymbol{d} \varepsilon\|>0,
$$

$\boldsymbol{d} \boldsymbol{\varepsilon}$ and $\boldsymbol{d} \boldsymbol{\sigma}$ being linked by the elasto-plastic relation. This product between the stress and strain tensor increments is called second order work and denoted $d^{2} W$. It is a scalar product between two vectors for engineering notations of $\boldsymbol{d} \boldsymbol{\varepsilon}$ and $\boldsymbol{d} \boldsymbol{\sigma}$. It means that, for a given loading direction, failure is detected if the material point cannot sustain any more a small perturbation [17]. This is viewed by Nova [25] as a "loss of controlability".. An example of a failure case specifically detected by this criterion is given by the undrained triaxial test on loose sand. In such a case the second order work is expressed: $d^{2} W=\boldsymbol{d q} \cdot \boldsymbol{d} \boldsymbol{\varepsilon}_{\text {axial }}$. In figure 3.b at the peak of $q:\|\boldsymbol{d} \boldsymbol{q}\|=0$ and $\left\|\boldsymbol{d} \varepsilon_{\text {axial }}\right\|=$ constant (if the test is kinematically controlled). Let us now consider the same test, but axially stress controlled. In such a case, failure is observed at the peak of $q$, that is to say when $d^{2} W$ becomes zero and for a stress state that has not reached yet the plastic limit criterion. The system is still subjected to strains, while no more energy is transferred to it. In other words, "...the deformation process continues 'on ones own'" [21].

Algebraically, the expression for the sufficient stability condition gives :

$$
\operatorname{det}\left(\boldsymbol{M}_{\boldsymbol{s}}\right)>0
$$

where $\boldsymbol{M}_{\boldsymbol{s}}$ is the symmetric part of the constitutive matrix $\boldsymbol{M}$. If $\boldsymbol{M}$ is symmetric, this condition is equivalent to the plastic limit criterion. Conversely, if the material is non associated, cases can appear when $\operatorname{det} \boldsymbol{M}_{\boldsymbol{s}}<0$ although $\operatorname{det} \boldsymbol{M}>0$ : it means that failure can happen inside the 'stability domain' defined by the plastic limit criterion [21].

This criterion has been successfully applied to geomechanical issues, including landslides initiation modelling $[26,27,23]$. Furthermore, as $(\operatorname{det} \boldsymbol{M}<0)$ and $(\operatorname{det} \boldsymbol{L}<0)$ bring directly $\left(\operatorname{det} \boldsymbol{M}_{\boldsymbol{s}}<0\right)$, it can be stated that the second order work criterion is a general condition able to detect instabilities leading to diffuse failures, as well as other failure types. Lastly, it has been proved that the annulation of $d^{2} W$ is linked to a burst of kinetic energy [19] for a small perturbation of the system.

For these reasons, it is obvious that the activation of a solid-fluid transition at failure is linked with the vanishing of $d^{2} W$. More accurately, an unstable material point can undergo an effective failure (and transition) if it is not constrained in the direction of its flow. In the present dealing of the transition, it is considered that failure and transition occurs at the material point directly from the vanishing of the second-order work. Nevertheless, the flow will only develop if it is not contained in a boundary model, that is to say if there a sufficient number of material points that have undergone the transition. This condition has been pointed 
in Lignon et al. [23] for slope stability analysis in which the global effective failure state is not achieved from the first negative values of $d^{2} W$, but from a significant number of unstable material points.

\subsection{Association of a solid and a fluid constitutive relation}

On one hand, soils are known to generally obey elasto-plastic constitutive relation, but after failure, no behaviour is generally proposed by the usual geomechanical models. On the other hand, granular suspensions, as mud-flows, are known to follow a viscous constitutive relation with a yield stress $[28,29]$, but rheological models classically suppose a rigid behaviour below this threshold.

Using the transition criterion of the second-order work, the new constitutive model can thus be based on both an elasto-plastic relation and a viscous law including a stress threshold. Starting from an initial elasto-plastic behaviour (that is to say from an in situ stable soil), the viscous strains are supposed to be suddenly activated from the failure state, and according to the Bingham model (which leads to a viscous flow of mud or debris). This transition model is to use for diffuse failure cases only, where a very sudden breakdown of the material structure is observed. This feature of instantaneousness is supported by the observation of sudden transitions of behaviour in many granular media (dry granular media as well as granular suspension), as mentioned in introduction.

As in many models of mud flow, the viscous flow stops (and the behaviour turns back elasto plastic) as soon as the stress state does not exceed anymore the yield stress (see section 2.4.2 for the 3D expression of this condition). At this stage, the material would turn back to elasto-plasticity with a new set of plastic parameters (due to the disorganisation of the granular structure). A simple elastic behaviour after the fluid $\rightarrow$ solid transition has been chosen for the moment.

On our model, the fluid $\rightarrow$ solid criterion (Bingham's yield stress) is different from the solid $\rightarrow$ fluid one (second order work) since soils elasto-plasticity behaviour, as well as granular suspensions viscosity one, strongly depend on the loading history (see for instance [3] for evidences of granular suspensions thixotropic behaviour). Given that the material starting state is not at all the same for one way of transition or another, there is, thus, no reason for the transition criterion to be the same.

The scheme of this association is given in figure 4 , in $1 \mathrm{D}$ and for any chosen elasto-plastic and viscous relation.

\subsection{Constitutive relations}

In this first study, among many available elasto-plastic relations and yield stress viscous ones, two specific models have been chosen namely Plasol and Bingham, keeping in mind that any other models could be used with the same approach.

\subsubsection{An elasto-plastic relation: Plasol}

The Plasol model has been developed in Liege University (Belgium) and great deal of information is given about that model in Barnichon [30]. Well suited to model a wide range of different soils, its main features are the following. 
Firstly, the plastic criterion is of Van Eekelen type [31]. Its representation in the 3D principal stress frame is a conical surface that depends on the three stress invariants, such as its base is not circular, as can be seen in figure 5b. It thus describes 2 important features of soils: the increase of strength with the confinement and the fewer resistance for extension stress loading (in the deviatoric plane) compare to compression stress loading. The equation of this criterion is:

$$
f=J_{2 \sigma}+m \cdot\left(J_{1 \sigma}-\frac{3 C}{\tan \varphi_{c}}\right)=0
$$

with:

- $J_{1 \sigma}=\operatorname{tr}(\boldsymbol{\sigma}), J_{2 \sigma}=\sqrt{\operatorname{tr}\left(\boldsymbol{s}^{2}\right)}, J_{3 \sigma}=\sqrt[3]{\operatorname{tr}\left(\boldsymbol{s}^{3}\right)}$ the three invariants of stress tensor $\boldsymbol{\sigma}$ $\left(\boldsymbol{s}=\boldsymbol{\sigma}-J_{1 \sigma} / 3 . \boldsymbol{I}\right.$ being the deviatoric part of tensor $\left.\boldsymbol{\sigma}\right)$,

- $C$ the cohesion,

- $\varphi_{c}$ the mobilized compression friction angle,

- $m$ a coefficient such as:

$$
m=a(1+b \sin (3 \theta))^{n}
$$

with:

- $\theta$ Lode's angle defined as: $\sin (3 \theta)=\sqrt{6}\left(J_{3 \sigma} / J_{2 \sigma}\right)^{3}$,

- $n$ a dimensionless parameter controlling the convexity of the criterion trace in the deviatoric plane,

- $a$ and $b$ defined as:

$$
b=\frac{\left(r_{c} / r_{e}\right)^{1 / n}-1}{\left(r_{c} / r_{e}\right)^{1 / n}+1} \quad \text { and } \quad a=\frac{r_{c}}{(1+b)^{1 / n}}
$$

where $r_{c}$ and $r_{e}$ are the compression and extension reduced radii define by $J_{3 \sigma} / J_{2 \sigma}$ for the triaxial test and expressed as (with the compression and extension friction angles $\varphi_{c}$ and $\varphi_{e}$ ):

$$
r_{c}=\frac{1}{\sqrt{3}}\left(\frac{2 \sin \varphi_{c}}{3-\sin \varphi_{c}}\right), \quad \text { and } \quad r_{e}=\frac{1}{\sqrt{3}}\left(\frac{2 \sin \varphi_{e}}{3+\sin \varphi_{e}}\right)
$$

Secondly, Plasol model allows hardening of the yield surface during the load. Generally, softening is not used with Plasol, which means that the plastic parameters increase during the plastic flow. The evolution of the mobilized plastic parameters (cohesion $C$, friction angle in extension and compression $\varphi_{e}$ and $\varphi_{c}$ ) is defined as follows:

$$
\begin{aligned}
& \varphi_{c}=\varphi_{c 0}+\frac{\left(\varphi_{c f}-\varphi_{c 0}\right) \varepsilon_{e q}^{p}}{B_{p}+\varepsilon_{e q}^{p}} \\
& \varphi_{e}=\varphi_{e 0}+\frac{\left(\varphi_{e f}-\varphi_{e 0}\right) \varepsilon_{e q}^{p}}{B_{p}+\varepsilon_{e q}^{p}} \\
& C=C_{0}+\frac{\left(C_{f}-C_{0}\right) \varepsilon_{e q}^{p}}{B_{c}+\varepsilon_{e q}^{p}}
\end{aligned}
$$


This evolution thus depends on the hardening parameters $B_{p}$ and $B_{c}$, and the history variable $\varepsilon_{e q}^{p}$, called the 'equivalent plastic strain', is defined as follows:

$$
\varepsilon_{e q}^{p}=\sqrt{\frac{2}{3} e_{i j}^{p} e_{i j}^{p}}
$$

with $e^{p}$ being the deviatoric part of plastic strain tensor $\varepsilon^{p}$.

According to the value reached by $\varepsilon_{e q}^{p}$, the plastic parameters vary between initial values with index ${ }_{0}$ - which define the elastic domain boundary, and final values -with index $f$ - which define the plastic limit surface, beyond which no equilibrium can be found.

Finally, Plasol can describe the typical non-associativity of soils, by a plastic potential (denoted $g$ ) which differs from $f$ (see equation 3 ). $g$ has the following form:

$$
g=J_{2 \sigma}+m^{\prime} \cdot\left(J_{1 \sigma}-\frac{3 C}{\tan \varphi_{c}}\right)
$$

where $m^{\prime}$ is similar to $m$, except that in its expression, the dilatancy angles $\left(\psi_{e}\right.$ in extension and $\psi_{c}$ in compression) replace the friction angles, with values generally lower by approximatively $20^{\circ}$. Dilatancy angles evolve in the same way as friction angle with hardening, but Plasol users only need to give the final values of $\psi_{e}$ and $\psi_{c}$, as the initial ones are deduced from Taylor's law [32]: the difference between the mobilized friction angle and the dilatancy one is constant. In total, 13 parameters are needed to describe an elasto-plastic soil, with isotropic elasticity and Plasol model.

\subsubsection{Bingham's viscous law}

Geomaterial suspensions are known to behave as viscous fluids with a yield stress (see [28] and [33] for mudflows, [34] for water-kaolinite artificial mixture, [35] for concrete...). Although, a non-linearity of the viscous relation has been also highlighted, the most important feature of the rheology is the presence of a minimum shear stress for the flow.

In a pure shear relation, which is suitable for a rheometer experiment but not for a real 3D issue, the constitutive relation is written as follows:

$$
\text { if } \quad|\tau|>s_{o}: \quad \dot{\gamma}=\left(\tau-s_{o} \operatorname{sgn}(\tau)\right) / \eta, \quad \text { else : } \quad \dot{\gamma}=0
$$

with $\eta$ the dynamic viscosity, $\tau$ the shear stress, $\dot{\gamma}$ the velocity gradient, and $s_{o}$ the yield stress. Function $x \rightarrow \operatorname{sgn}(x)$ returns the sign of scalar $x$.

In three dimensions, and supported by the expression of Duvaut and Lions [36] and Balmforth and Craster [37], it can be expressed this way:

$$
\text { if } \quad J_{2 \sigma}>s_{o}: \quad \dot{e}_{i j}=\frac{1}{2 \eta}\left(s_{i j}-s_{o} \frac{s_{i j}}{J_{2 \sigma}}\right)=\frac{J_{2 \sigma}-s_{o}}{2 \eta} \cdot \frac{s_{i j}}{J_{2 \sigma}}, \quad \text { else }: \quad \dot{e}_{i j}=0
$$

$\boldsymbol{s}$ and $\dot{\boldsymbol{e}}$ are respectively the deviatoric stress and strain rate tensors, $J_{2 \sigma}$ and $J_{2 \dot{\varepsilon}}$ are the second invariant of stress and strain rate tensors. The direction of the yield stress is thus given by the direction $s_{i j} / J_{2 \sigma}$ of the deviatoric stress tensor.

To conclude, the characteristic surfaces of the global 3D transition model are presented in figure 5, for realistic parameters in mudflows: $\varphi_{c}=\varphi_{e}$ vary from 6 to $28^{\circ}, C$ varies from 1 to $10 \mathrm{kPa}$ and $s_{o}=2 \mathrm{kPa}$. These surfaces are (in the principal stress space): the plastic limit criterion, the elastic limit surface, and the viscous threshold. It must be recalled that failure can occur inside the plastic limit surface. 


\section{Using Finite Element Method with Lagrangian Inte- gration Points (FEMLIP)}

\subsection{Basis of the method and advantages for landslides modelling}

As seen in introduction, one of the difficulty to simulate landslide lays in the ability of numerical methods to be well suited both for history dependent behaviours and high levels of displacements. Let us see now how FEMLIP method fits to these requirements.

Firstly, according to its name, FEMLIP is based on Finite Element methods (FEM), with a full continuous approach and a discretization of the whole equilibrium problem on a finite number of equations at nodes. But FEMLIP, as the Particle In Cell (PIC) method from which it originates [38], has the specificity to work with a double discretization of the domain, instead of the unique space partition in elements as in FEM [39, 40].

First, the domain of study is partitioned into elements in order to get the nodal equations. In FEMLIP, like in a Eulerian FEM, the mesh is fixed. It only stands for a computational grid which does not hinder the integration process (and thus the convergence of the computation), since it is not strained with the material. This last point is essential to take into account the high degree of deformations in flow models. Secondly, the material itself is discretized in what is usually called 'particles' or 'material points'. It must be emphasized that they do not represent physical grains as in the discrete element method (there is no contact interaction between them). They only stand for numerical supports that carry material information during the advection or the deformation of the different bodies. In this way, the material variables (such as plastic strains, material properties, etc.) are known with accuracy over the domain and through the whole computation. It thus enables the handling of history dependant behaviour and an accurate tracking of different coexisting materials. This principle is illustrated in figure 6.

The two discretization systems are disconnected, since the material points are moving across the mesh, according to the material kinematic field resulting from the resolution of equilibrium equations. The link between the two is temporarily re-established at each computational increment by using the particles as integration points, each one being associated with specific coordinates and numerical weight that depend on the current configuration. These two sets of data thus need to be re-computed at every step [40].

Finally, FEMLIP can handle both a precise tracking of the material variables (illustrated in figure 6) and a high level of deformations, and thus that it is well suited for landslide modelling. Only few continuum methods are able to respond to this double requirement: one could mention SPH method which is developed in this way (see introduction), or Material Point Method [44] which also comes from PIC methods (but with a different integration process, see $[40])$.

\subsection{Formulation of the problem}

\subsubsection{Governing equation}

The governing equation of the formulation is Navier-Stokes equation of momentum conservation:

$$
\operatorname{div} \boldsymbol{\tau}-\operatorname{grad} p+\boldsymbol{f}=0
$$


in which $\boldsymbol{\tau}$ is the deviatoric stress tensor, $p$ is the pressure and $\boldsymbol{f}$ is the volumetric resulting force vector. In our approach, the inertial terms are not considered.

The problem is always based on viscous relation and the resolution matrix is defined with viscous shear and bulk parameters. If visco-elastic behaviour is considered, effective viscous parameters are defined along the time increments, taking into consideration the relaxation time defined as the ratio between the viscosity and the elastic shear modulus [40]. Quasielastic behaviour can be achieved for a suitable choice of the time step and of the viscous parameters which become, then, only numerical variables.

For a pure viscous behaviour, the detailed formulation of the system of equation on nodes is similar to the one of a classical finite element method. In case of visco-elastic behaviour, it is presented in Moresi et al. [40].

The FEMPIL-based code used for the resolution is called Ellipsis.

\subsubsection{Hypothesis}

In addition to consider static solution for the resolution, other hypothesis are made in the resolution.

First, plane strain conditions are considered in Ellipsis.

Besides, the code is still in development concerning hydro-mechanical coupling, and at the moment only purely mechanical or thermo-mechanical problems can be solved.

Lastly, and this point is directly due to the principle of FEMLIP method, because the mesh position has to be known in advance for the computation, normal stress cannot be applied on the boundaries. That implies that free surface models (which corresponds to a zero stress application on a frontier) can only be described by considering -in addition to the material of interest- a filling material, inside the fixed boundary model, with negligible parameters (usually chosen as viscous). It is equivalent to say that it is not only the material on interest which must be discretised but all the domain in which it initially stands and will flow in.

\subsubsection{Implementation of the transition model}

As it was recalled, Ellipsis was first used to model fluid problems of geophysics, as mantel convection for instance. Many viscous constitutive relations (linear as well as non linear) are thus implemented in the code. Notably, non linear expression of the viscosity has been considered to describe Bingham threshold in the context of fresh concrete modelling [41, 35]. Besides, developments have been made in order to take into account visco-elastic constitutive laws (based on a Maxwell model written in 3D). This enhancement has been used to model, for example, lithospheric deformation [45], or more generally geological layers folding [40]. Very lastly, the code has been adapted for visco-elasto-plastic models, with an adaptive plug of the plastic law [42].

Concerning the implementation of the second order work criterion, the modifications were few in the code. It has consisted in computing, for each material point and at each step, the normalized form of the second order work expressed this way:

$$
d W_{n}^{2}=\frac{d \sigma_{i j} d \varepsilon_{i j}}{\|\boldsymbol{d} \boldsymbol{\sigma}\|\|\boldsymbol{d} \varepsilon\|}
$$

This quantity is not calculated for all loading directions but only for the current loading path, since the loss of stability is more looked for than a unconditional stable state. Besides, in order to be sure that the system is unstable, the transition is activated as soon as $d W_{n}^{2}<10^{-3}$. 
Finally, at a given step $n$, the stress and strain tensor increments for the calculus of $d W_{n}^{2}$ correspond to the quantity calculated between step $n-2$ and step $n-1$. The second order work is thus an explicit variable, but it can be considered only few variations of it from step to step, for a sufficiently slow loading.

Concerning the Bingham 3D threshold $s_{o}$, the implementation scheme for visco-elastoplasticity followed in [42] has been slightly modified to take into account a viscous deviatoric strain rate that is now function of $s_{o}$ (as expressed in equation 6) In the same way as Cuomo et al. [42] established a new force term depending on the plastic strain tensor, here, the writing of the equilibrium makes appear -if $J_{2 \sigma}$ oversteps $s_{o^{-}}$a new force term depending on this threshold.

\section{Presentation of the boundary model studied}

\subsection{Geometry and boundary conditions}

The first model studied with the transition constitutive model implemented into Ellipsis code, is a simplified heuristic problem.

In order to consider the propagation of a flow from an unstable geomaterial, a simple approach is to consider (i) a mass of soil located on the top of a slope and (ii) a lateral extension of the model which is not too large in order not to have a too thin layer of flow with comparison with its length (which would be much more difficult to discretize). Studying the flow arrest against a structure is also a major point of interest.

Given these considerations, the geometry has been chosen to be the same as the one of Preisig and Zimmermann [46], who simulated a small scale viscous mudflow against an obstacle (but not the initiation phase). The model is made of a $27^{\circ}$ slope, a metric square sample of soil at its top and a downstream obstacle (see figure 7). The boundary conditions have also been chosen identical: fixed nodes on the left boundary, and free slip in the others. The similarity of the two models make possible to later compare our results to theirs.

Finally, the mesh is formed by quadrilateral bi-linear elements: 72 along x-axis and 48 along $\mathrm{y}$-axis which follows the model boundaries (axes are shown in figure 7 ).

\subsection{Determination of the parameters}

The parameters of this heuristic model have to be chosen as close as possible of those of a real mud-flow. For this purpose, bibliography about Campania mud-flows has been carefully analysed. In this Italian region, volcanic ashes of the slope cover layers regularly lose its stability under intense rainfalls (Sarno and Quindici mudslide is an example of such event). Indeed, the saturation increase induces a reduction of the over-strength allowed by the suction, which reaches 10 to $70 \mathrm{kPa}$ in those deposits [47]. Moreover, it has been demonstrated that those soils have been subjected to a diffuse failure [48] which corresponds to the application field of our model.

Let us recall that, as seen in section 2, the global transition model needs 15 parameters to be run (13 elastoplastic ones and 2 viscous ones) and besides, the density of the material needs to be known.

Thanks to many publications $[48,49,50]$, it is possible to get, for the ashes, reliable values of elastic parameters $(E=5 \mathrm{MPa}$ and $\nu=0.29)$, natural density $\left(\gamma_{n a t}=16 \mathrm{KN} / \mathrm{m}^{2}\right)$ and drained friction angle at plastic failure $\left(\varphi^{\prime}=38^{\circ}\right)$. Drained cohesion $C^{\prime}$ is also given by Crosta and 
Negro [50] -about a few $\mathrm{kPa}$ - but it is indeed reasonable to consider a high variability of the apparent cohesion with the level of saturation. As no hydraulic phase is considered in the present modelling, this variable apparent cohesion is considered as an entry parameter and it is denoted $C$. Besides, it is geometrically estimated that, to have no intersection between elastic limit surface and plastic limit surface in the principal stresses space, $C_{o}$ and $\varphi_{o}$ (at the elastic limit) have to be 5 times smaller than $C_{f}$ and $\varphi_{f}$ (at the plastic limit criterion). Parameter $n$ of Plasol law is -in practice- usually chosen of -0.229 to get a reasonable convexity of the plastic criterion. Finally, it is assumed that friction and dilatancy angles are the same in compression and in extension and that hardening parameters $B_{p}=0.01$ and $B_{c}=0.02$ are equal to those chosen by Lignon et al. [23] for Petacciato (Italy) landslide model, where fine granular materials (mostly silts) are also involved.

Concerning viscous parameters, to the author's knowledge no data are available for viscosity and stress threshold for Campania flowslides (the existing numerical simulations of the flows use, for the most part, depth integrated models without the need for viscous parameter). Nevertheless, a more general bibliography about mudflows studied with Bingham's model reveals a range of viscosity between 30 and $1000 \mathrm{~Pa}$.s and a range of stress threshold between 0,1 and $12 \mathrm{kPa}[51,52,53,54]$. It is important to notice that those orders of magnitude cannot be defined more precisely because of the influence on the viscous parameters of the soil nature and the water content. In those ranges, quite low values of $\eta$ and $s_{o}$ are arbitrarily chosen (150 Pa.s and $1.5 \mathrm{kPa}$ ) and, besides, it is supposed that the viscous bulk modulus is infinite (no viscous volumetric deformation).

In addition, an isotropic linear elastic constitutive relation is chosen for the obstacle, with $E=500 \mathrm{kPa}$ and $\nu=0.29$. Finally, let us recall that negligible viscous parameters are attributed to the surrounding material (figured without colour in figure 7). The whole physical parameters are finally gathered in table 1 .

\subsection{Applied load}

Although the most critical triggering factor for diffuse failure in soils appears to often be an hydraulic condition like rain or water table rising, such a coupled hydro-mechanical problem cannot be simulated presently with Ellipsis code. A simple increase of gravity is thus considered here, with a final value of $9.8 \mathrm{~m} / \mathrm{s}^{2}$ achieved in 50 increments.

Various simulations are performed, focusing first on the loss of stability and then on the propagation stage and the arrest.

\section{Results and discussion}

\subsection{Initiation stage and influence of the cohesion on stability}

In this first stage, no obstacle is considered down the slope. As said previously, it is expectable that the apparent cohesion $C$ of the soil varies according to the suction due to the incomplete saturation. It is thus of importance to estimate is influence on the stability that is why the model is run with $C$ varying between values of $C_{o}=1 \mathrm{kPa}$ and $C_{f}=5 \mathrm{kPa}$ up to higher values of $C_{o}=4 \mathrm{kPa}$ and $C_{f}=20 \mathrm{kPa}$.

For the different simulations, the gravity $g$ at which failure state and viscous behaviour are achieved for at least one material point (according respectively to the second-order work criterion and to Bingham threshold) is determined. The results are presented in figure 8, on 
which the first plastic deformation, the first failure state, and the first viscous behaviour are reported in function of the gravity reached and the cohesion considered (the cohesion presented on the abscissa corresponds to the plastic limit value, $C_{f}$ ).

One can see first that the gravity level for a first plastic deformation, failure or viscous behaviour consistently increases with the cohesion. For a cohesion greater or equal to $C_{o}=4$ and $C_{f}=20 \mathrm{kPa}$, the load until $g=9.8 \mathrm{~m} / \mathrm{s}^{2}$ still leads to plastic strains but not to failure nor to viscous behaviour (these last are reached for a value of $g$ of about $9.9 \mathrm{~m} / \mathrm{s}^{2}$ ). This hardening range of $C$ is thus the minimum for stability.

Besides, it can be noticed, that, for low values of cohesion (as $C_{o}=1$ and $C_{f}=5 \mathrm{kPa}$ ), the appearance of viscous behaviour does not exactly coincide with the failure. This can be explained by the proper formulation of the transition model: according to section 2 , the viscosity is activated at one material point if both the failure state is reached and the yield stress is overstepped by the second stress invariant. For very small values of cohesion it occurred that the Bingham yield stress is a more limiting criterion than the failure one. That is just a numerical aspect due to an unsuitable choice of the parameters for this simulation. In real cases, if a stress tensor is not sustained any-more by a granular structure it is very likely that this material would not resist to the flow under the second stress invariant of such tensor.

Finally, the spatial distribution of failure and viscous zones is depicted in figure 9 for a cohesion varying between $C_{o}=2$ and $C_{f}=10 \mathrm{kPa}$ (according to figure 8 , this range is high enough to have consistency between the failure and the viscous behaviour and low enough to reach unstable states in the soil). Indeed, it can be checked that failure induces quasi-directly a viscous behaviour since the 2 zones are almost identical (the few parts where failure is achieved but not viscous behaviour are explained by the Bingham threshold). Besides, it must be underlined that failure affects the soil with a diffuse pattern: that matches the framework of our model.

\subsection{Propagation stage and influence of the yield stress on the arrest}

\subsubsection{Flow phase modelling}

First, for the same parameters and $C=2$ to $10 \mathrm{kPa}$, the rest of the computation -without obstacle- leads to the configuration presented in figure 10. Concretely, the modelling of an obstacle can thus aim to protect the zone situated between $x=2.5$ and $x=3 \mathrm{~m}$.

The results obtained, in terms of configuration, are shown in figure 11, on which the areas of solid-like and fluid-like behaviours can be distinguished. The solid-like behaviour prevails before the initiation of the flow (it is elasto-plastic in this first stage) and after its arrest (it is considered elastic in first approximation as seen in section 2). The viscous fluid behaviour obviously prevails during the flow.

The diffuse failure stage (figure 11a) is the same as previously. During the general flow stage (figure 11b), one can see that the behaviour of the whole bulk of soil is fluid like, except in the top corner which undergoes less shear stress. At the specific increment shown in figure 11b, the velocity of the front has reached, in less than one second, about $5 \mathrm{~m} / \mathrm{s}$ in the slope direction. This value is realistic compared to the velocity usually observed in landslide of the same type, which is around few meters/second. Nevertheless, with such a fast increase of velocity, one can call into question the disregarding of inertial effects. Some responses are given about that point in section 5.3.

At the first contact (figure 11c), the flow starts to retrieve a solid behaviour from its 
front. Finally, in a last stage (figure 11d), the soil has an entire solid behaviour except a last remaining band where the behaviour is still viscous because of the large shear stress induced by the geometry of the obstacle. This band separates an upper zone where, due to the viscous shear, there is still a little velocity (around $12 \mathrm{~cm} / \mathrm{s}$ at this step), and a lower zone, against the obstacle foot, where velocity is closer to zero (around $4 \mathrm{~cm} / \mathrm{s}$ ). This last corresponds to a "dead zone" which is the first to stop and whose geometrical characterising is essential for protection works sizing $[55,56]$. Once the flow has completely stopped, the x-displacement $D_{x}$ of the top of the obstacle (point $\mathrm{P}$ situated in figure 7) is about $3 \mathrm{~cm}$, while the value of the second stress invariant $J_{2 \sigma}$ at its base (point P' situated in figure 7 ) is about $14 \mathrm{kPa}$.

Now, let us focus in this interaction with this protection work, and determine the influence of the viscous yield stress on $D_{x}(P)$ and $J_{2 \sigma}\left(P^{\prime}\right)$.

\subsubsection{Parametric study to establish $s_{o}$ influence}

Four values are considered, in addition to $s_{o}=1.5 \mathrm{kPa}$ already tested: $0.1,0.5,1$ and $2 \mathrm{kPa}$.

The resulting configurations after 1.1 second can be compared at figure 12 for cases $s_{o}=0.1$, 1.0 and $2.0 \mathrm{kPa}$. It appears, in a consistent manner, that the lower the threshold, the later the flow stops and the further it tends to spread. For the two lowest values of $s_{o}(0.1$ and 0.5 $\mathrm{kPa}$ ), the flow finally oversteps the obstacle, inducing a clear bending of it (see figure 12(a) for $s_{o}=0.1 \mathrm{kPa}$ ).

Evolutions of $J_{2 \sigma}\left(\mathrm{P}^{\prime}\right)$ (base of the obstacle) and $D_{x}(\mathrm{P})$ (top of the obstacle) have been extracted and are presented in figure 13. They have been chosen to be plotted along time, which is meaningful only during the viscous flow which is focused on (but must not be considered for the elasto-plastic initiation phase). Each of the two data is picked up for a material point that naturally follows the structure deformation.

Firstly, mere observations can be made. The lower $s_{o}$, the higher $J_{2 \sigma}$ and $D_{x}$ result, which is consistent regarding -with a diminution of the yield stress- the mass increase reaching the obstacle. Besides, a diminution of $s_{o}$ reduces the time of the first contact with the structure (figure 13(a)), this time being defined as the first significant increase of $D_{x}$ (a slight displacement increase appears previously, due to a negligible effect of the surrounding material).

A finer analysis of graph 13 (b) allows to notice that the final value of $J_{2 \sigma}$ tends to stabilize with the decrease of $s_{o}$ : there is a great difference between the final curves for $s_{o}=2$ and 1.5 $\mathrm{kPa}$, but only a low one between $s_{o}=0.1$ and $0.5 \mathrm{kPa}$. This can be explained by the fact that the maximum volume available behind the obstacle is constant. Thus, for a sufficiently low yield stress, the mass accumulation against the obstacle (which is determinant for the stresses applied on it) is not limited by a solidification along the slope, but only by the overstepping of the constant height $H$ of the structure.

Finally, for low values of $s_{o}$, it must also be distinguished in both graphs a phase of rapid increase of $D_{x}$ and $J_{2 \sigma}$, between 0.4 and $0.6 \mathrm{~s}$, and a phase where this increase is more limited, after $0.6 \mathrm{~s}$. Comparing this timing to the configuration evolution, it appears that the first phase corresponds to the first contact with the obstacle, whereas the second one corresponds to the filling of the volume behind the obstacle. This distinction gets smoother for higher values of $s_{o}$ which may be interpreted by the fact that, for a flow of a limited extent, the first contact and the reservoir filling are not such distinct phases.

In this section the validity of the static hypothesis has been questioned. That is why, in a last point a comparison has been made with some dynamical results obtained for this boundary value model by [46]. 


\subsection{Force on the obstacle}

As explained previously, Preisig and Zimmermann's model [46] and ours have exactly the same initial geometry and boundary conditions. The numerical method used by these authors is close to the Particle Finite Element Method [57], with -at each step- a re-meshing of the domain and a free surface tracking. It is not clear if this method would be able to describe solid behaviour but in the model presented, no initiation phase (with elasto-plasticity and failure) is described, and no deformation of the obstacle is modelled (indeed the structure is only considered as part of the domain boundaries, fixed in space). The nodal unknowns are velocity and acceleration such as the resolution takes into consideration inertial effects, contrary to our computation.

Preisig and Zimmermann made a first computation for a single phase with a density of $7.50 \mathrm{kN} / \mathrm{m}^{3}$ and a viscosity of 50 Pa.s (no yield stress is considered) while the load only consists in applying the gravity $g$ in one step. In a second time, they focused on the solid and fluid phase repartition with a second computation where the phases are only distinguished by distinct values of viscosity and density. The resulting force on the obstacle surface is determined for both computations. From our part, the same computation as previously is performed, but by changing $\eta$ and $\gamma$ according to Preisig and Zimmermann's parameters and setting $s_{o}=0$. As the obstacle boarders do not necessarily coincide with the mesh nodes, the resulting force is computed by considering the equilibrium over the structure area. Given that, this last has no density, the whole force on it is applied only on its surfaces. The sum of the nodal forces at the bottom boundary of the obstacle (which are reaction forces), is thus the opposite of the resulting forces applied by the flow on the structure.

On one hand, the configuration obtained by Preisig and Zimmermann after $1 \mathrm{~s}$ as well as their calculus of the force temporal evolution are presented respectively in figure 14 and 15(a). On the other hand, the force versus time we obtained is presented in figure 15(b).

First, in figure 14, one cannot miss the jump of the flow over the structure, which is not observed in our results, these last being very similar for these parameters than those presented in figure 12(a). This is a dynamical effect that our computation was not able to reproduce.

Secondly, comparing the two force evolutions versus time, different aspects can be pointed out. On one hand, it can be noticed that the contact with the obstacle -when the force first increases- is achieved at the same time (around $0.5 \mathrm{~s}$ ) for both models. Moreover, at the end of the computation, the two values are tending to be very close one another, between 1700 and $1800 \mathrm{~N}$. But on the other hand, it is clear that the force evolution between 0.5 and $0.7 \mathrm{~s}$ are strongly diverging. A force peak is obtained by Presig and Zimmermann, while we do not have such high response. This peak, which corresponds to the dynamical impact force, is another manifestation of the inertial effects that our code, in its actual development state, is missing.

In conclusion, our computation has been shown to be reliable (first contact time and final force are confirmed), but, as expected with the quasi-static hypothesis done in Ellipsis code, some significant effects are disregarded in our computation in the case of quite high velocities (here, let us recall they are of few meters/s).

\section{Conclusion and prospects}

In conclusion, this work has achieved various objectives.

First of all, the new constitutive relation successfully describes the drastic evolution of insitu soils behaviour that can be observed during a mudflow development. Besides, the choice 
of suitable elasto-plastic and viscous constitutive relations makes possible to take into account both soils and mud suspensions specific mechanical features.

In addition to describe these contrasting behaviours, the new model takes into account the transition with a criterion, the second order work, which is mechanically consistent since it can detect any material point divergence instability. In particular, all cases of soil diffuse failures (which can lead to solid-fluid transition) are thus dealt with.

Consequently, a first boundary model performed with the new constitutive relation and using FEMLIP method, is able to describe the consistent evolution of a mudflow during its initiation, its propagation and its arrest. The efforts made to link, as far as possible, our results in the flowing phase with quantitative numerical data made appeared good agreement with those last, since:

- the values of velocity obtained are realistic with regard to real natural flow ones,

- the time of the first flow/obstacle contact is similar to the one got by Preisig and Zimmermann,

- the force developed against the structure considered is, in a static state (that is to say waiting enough after the flow/obstacle first contact), very close to Preisig \& Zimmermann's one.

It must be underlined that FEMLIP method proved -once more- its efficiency concerning complex behaviour dealing, and is a good candidate for landslide whole modelling.

Given this high modelling potential obtained by associating the new proposed model with a method such as FEMLIP, many prospects can be considered, notably in natural risks domain (landlisdes, flowslides, avalanches ...).

Some points still are to be further investigated to enhance the application fields of our modelling, as the dynamical aspects for fast flows (they influence the impact forces as seen in section 5), and the hydro-mechanical coupling for rainfall triggered losses of stability.

\section{References}

[1] H.M. Jaeger and S.R. Nagel. Granular solids, liquids, and gases. Reviews of Modern Physics, 68(4):1259-1273, 1996.

[2] P.G. de Gennes. Granular matter: a tentative view. Reviews of Modern Physics, 71(2):S374-S382, 1999.

[3] P. Coussot, N. Roussel, S. Jarny, and H. Chanson. Continuous or catastrophic solid-liquid transition in jammed systems. Physics of Fluids, 17:011704, 2005.

[4] P. Coussot, J.S. Raynaud, F. Bertrand, P. Moucheront, J.P. Guilbaud, H.T. Huynh, S. Jarny, and D. Lesueur. Coexistence of liquid and solid phases in flowing soft-glassy materials. Physical Review Letters, 88(21):218301, 2002.

[5] F. Radjai, D.E. Wolf, M. Jean, and J.J. Moreau. Bimodal character of stress transmission in granular packings. Physical review letters, 80(1):61-64, 1998.

[6] S. Deboeuf, O. Dauchot, L. Staron, A. Mangeney, and J.P. Vilotte. Memory of the unjamming transition during cyclic tiltings of a granular pile. Physical Review E, 72(5):051305, 2005. 
[7] L. Staron, J.P. Vilotte, and F. Radjai. Preavalanche instabilities in a granular pile. Physical review letters, 89(20):204302, 2002.

[8] I.S. Aranson and L.S. Tsimring. Continuum theory of partially fluidized granular flows. Physical Review E, 65(6):061303, 2002.

[9] N. Huang. Rhéologie des pâtes granulaires. PhD thesis, Université Paris 6, 2006.

[10] P. Coussot, Q.D. Nguyen, H.T. Huynh, and D. Bonn. Viscosity bifurcation in thixotropic, yielding fluids. Journal of Rheology, 46(3):573-589, 2002.

[11] P. Jop, Y. Forterre, and O. Pouliquen. A constitutive law for dense granular flows. Nature, 44:727-730, 2006.

[12] M. Pastor, D. Manzanal, J.A. Fernández Merodo, P. Mira, T. Blanc, V. Drempetic, M.J. Pastor, B. Haddad, and M. Sánchez. From solids to fluidized soils: diffuse failure mechanisms in geostructures with applications to fast catastrophic landslides. Granular Matter, $12: 211-228,2010$.

[13] D. Laigle, P. Lachamp, and M. Naaim. Sph-based numerical investigation of mudflow and other complex fluid flow interactions with structures. Computational Geosciences, 11(4):297-306, 2007.

[14] H.H. Bui, R. Fukagawa, K. Sako, and S. Ohno. Lagrangian meshfree particles method (sph) for large deformation and failure flows of geomaterial using elastic-plastic soil constitutive model. International journal for numerical and analytical methods in geomechanics, 32(12):1537-1570, 2008.

[15] D. Bigoni and G. Noselli. Experimental evidence of flutter and divergence instabilities induced by dry friction. Journal of Mechanics and Physics of Solids, 59,10:2208-2226, 2001.

[16] HDV Khoa, IO Georgopoulos, F. Darve, and F. Laouafa. Diffuse failure in geomaterials: Experiments and modelling. Computers and Geotechnics, 33(1):1-14, 2006.

[17] A. Daouadji, F. Darve, H. Al Gali, P.Y. Hicher, F. Laouafa, S. Lignon, F. Nicot, R. Nova, M. Pinheiro, F. Prunier, L. Sibille, and R. Wan. Diffuse failure in geomaterials: Experiments, theory and modelling. International Journal of Numerical and Analytical Methods in Geomechanics, 35(16):1731-1773, 2011.

[18] F. Nicot and F. Darve. Diffuse and localized failure modes, two competing mechanisms. International Journal for Numerical and Analytical Methods in Geomechanics, 35,5:586$601,2011$.

[19] F. Nicot, A. Daouadji, F. Laouafa, and F. Darve. Second-order work, kinetic energy and diffuse failure in granular materials. Granular Matter, 13,1:19-28, 2011.

[20] J.W. Rudnicki and J. Rice. Conditions for the localization of deformation in pressure sensitive dilatant materials. International Journal of Solids and Structures, 23:371-394, 1975 . 
[21] F. Darve, G. Servant, F. Laouafa, and H.D.V. Khoa. Failure in geomaterials: continuous and discrete analysis. Computer Methods in Applied Mechanics and Engineering, 2729,193:3057-3085, 2004.

[22] J.R. Rice. Inelastic constitutive relations for solids: an internal-variable theory and its application to metal plasticity. Journal of the Mechanics and Physics of Solids, 19:433455, 1971.

[23] S. Lignon, F. Laouafa, F. Prunier, HDV Khoa, and F. Darve. Hydro-mechanical modelling of landslides with a material instability criterion. Geotechnique, 59(6):513-524, 2009.

[24] R. Hill. A general theory of uniqueness and stability in elasto-plastic solids. Journal of the Mechanics and Physics of Solids, 6:236-249, 1958.

[25] Roberto Nova. Controllability of the incremental response of soil specimens subjected to arbitrary loading programmes. Journal of the Mechanical behavior of Materials, 5(2):193202, 1994.

[26] F. Darve and F. Laouafa. Instabilities in granular material and application to landslides. Mechanics of Cohesive-Frictional Materials, 5:627-652, 2000.

[27] F. Laouafa and F. Darve. Modelling of slope failure by material instability mechanism. Computers and Geotechnics, 29:301-325, 2002.

[28] A. Daido. On the occurence of mud-debris flow. Bulletin of the Disaster Prevention Research Institute, Kyoto Univ, 21:109-135, 1971.

[29] Philippe Coussot and Stéphane Boyer. Determination of yield stress fluid behaviour from inclined plane test. Rheologica acta, 34(6):534-543, 1995.

[30] J.D. Barnichon. Finite Element Modeling in Structural and Petroleum Geology. PhD thesis, Université de Liège, 1998.

[31] H.A.M. VanEekelen. Isotropic yield surface in three dimensions for use in soil mechanics. International Journal for Numerical and Analytical Methods in Geomechanics, 4:89-101, 1980.

[32] D. Taylor. Fundamentals of Soil Mechanics - London Wiley, 1948.

[33] P. Coussot and J.M. Piau. On the behavior of fine mud suspensions. Rheological Acta, $33: 175-184,1994$.

[34] P. Coussot, S. Proust, and C. Ancey. Rheological interpretation of deposits of yield stress fluids. Journal of Non-Newtonian Fluid Mechanics, 66:55-70, 1996.

[35] N. Roussel. Rheology of fresh concrete: from measurements to predictions of casting processes. Materials and Structures, 40(10):1001-1012, 2007.

[36] G. Duvaut and J.L. Lions. Transfert de chaleur dans un fluide de bingham dont la viscosité dépend de la température. Journal of Funtional Analysis, 11:93-110, 1972.

[37] N.J. Balmforth and R.V. Craster. A consistent thin layer theory for bingham plastics. Journal of non-newtonian fluid mechanics, 841:65-81, 1999. 
[38] F.H. Harlow. The Particle-In-Cell Computing Method for Fluid Dynamics in Fundamental Methods in Hydrodynamics. B. Lader and S. Fernbach and M. Rotenberg, 1964.

[39] L. Moresi and V.S. Solomatov. Numerical investigation of 2d convection with extremely large viscosity variations. Physics of Fluids, 7:2154, 1995.

[40] L. Moresi, F. Dufour, and H.B. Mühlhaus. A lagrangian integration point finite element method for large deformation modeling of viscoelastic geomaterials. Journal of Computational Physics, 184(2):476-497, 2003.

[41] F. Dufour and G. Pijaudier-Cabot. Numerical modelling of concrete flow: homogeneous approach. International journal for numerical and analytical methods in geomechanics, 29(4):395-416, 2005.

[42] S. Cuomo, N. Prime, AL. Iannone, F. Dufour, L. Cascini, and F. Darve. Large deformation femlip drained analysis of a vertical cut (accepté). Acta Geotecnica ASCE, 1:1, 2012.

[43] H.B. Mühlhaus, F. Dufour, L. Moresi, and B. Hobbs. A director theory for visco-elastic folding instabilities in multilayered rock. International Journal of Solids and Structures, 39(13):3675-3691, 2002.

[44] D. Sulsky and H.L. Schreyer. Axisymmetric form of the material point method with applications to upsetting and taylor impact problems. Computer Methods in Applied Mechanics and Engineering, 139(1):409-429, 1996.

[45] C. O'Neill, L. Moresi, D. Müller, R. Albert, and F. Dufour. Ellipsis 3d: A particle-in-cell finite-element hybrid code for modelling mantle convection and lithospheric deformation. Computers and Geosciences, 32(10):1769-1779, 2006.

[46] M. Preisig and T. Zimmermann. Two-phase free-surface fluid dynamics on moving domains. Journal of Computational Physics, 229(7):2740-2758, 2010.

[47] L. Olivares and L. Picarelli. Occurrence of flowslides in soils of pyroclastic origin and consideratoins for landslide hazard mapping. In Proceedings of 14th South-East Asian Conference, HongKong, pages 881-886, 2001.

[48] L. Olivares and L. Picarelli. Suceptibility of loose pyroclastic soils to static liquefaction : some preliminary datas. In Kühne M, Einstein HH, Krauter E, Klapperich H, Pöttler $\mathrm{R}$, editor, Proceedings of International Conference on Landslides - Causes, Impacts and Countermeasures, Davos, pages 75-85, 2001.

[49] L. Cascini, S. Cuomo, M. Pastor, and G. Sorbino. Modeling of rainfall-induced shallow landslides of the flow-type. Journal of geotechnical and geoenvironmental engineering, $136: 85,2010$.

[50] GB. Crosta and P. Dal Negro. Observations and modelling of soil slip-debris flow initiation processes in pyroclastic deposits: the sarno 1998 event. 2003.

[51] J.K. Jeyapalan, J.M. Duncan, and H.B. Seed. Investigation of flow failures of tailing dams. Journal of Geotechnical Engineering ASCE, 109:172-189, 1983. 
[52] M. Pastor, J.A. Fernández Merodo, M.I Herreros, P. Pira, E. González, B. Haddad, M. Quecedo, L. Tonni, and V. Drempetic. Mathematical, constitutive and numerical modelling of catastrophic landslides and related phenomena. Rock Mechanics and Rock Engineering, 41(1):85-132, 2008.

[53] K. Soga. Failure observed in landslides. Presentation in Alert Worshop, 2011.

[54] F.V. De Blasio, A. Elverhoi, D. Issler, C.B. Harbitz, P. Bryn, and R. Lien. Flow models of natural debris flows originating from overconsolidated clay materials. Marine Geology, 213:439-455, 2004.

[55] B. Chanut, T. Faug, and M. Naaim. Time-varying force from dense granular avalanches on a wall. Physical Review E, 82(4):041302, 2010.

[56] T. Faug, R. Beguin, and B. Chanut. Mean steady granular force on a wall overflowed by free-surface gravity-driven dense flows. Physical Review E, 80(2):021305, 2009.

[57] SR Idelsohn, E. Oñate, and F.D. Pin. The particle finite element method: a powerful tool to solve incompressible flows with free-surfaces and breaking waves. International Journal for Numerical Methods in Engineering, 61(7):964-989, 2004. 
Table 1: Synthesis of chosen parameters

\begin{tabular}{|l|c|c|c|c|c|c|c|c|}
\cline { 2 - 9 } \multicolumn{1}{c|}{} & $\begin{array}{c}\gamma_{\text {nat }} \\
\left(\mathrm{kN} / m^{3}\right)\end{array}$ & $\begin{array}{c}\mathrm{E} \\
(\mathrm{MPa})\end{array}$ & $\nu$ & $\begin{array}{c}C^{\prime} \\
(\mathrm{kPa})\end{array}$ & $\begin{array}{c}\varphi_{e}^{\prime}=\varphi_{c}^{\prime} \\
\left({ }^{\circ}\right)\end{array}$ & $\begin{array}{c}\psi_{e}=\psi_{c} \\
\left({ }^{\circ}\right)\end{array}$ & $\begin{array}{c}\eta \\
(\text { Pa.s })\end{array}$ & $\begin{array}{c}s_{o} \\
(\mathrm{kPa})\end{array}$ \\
\hline Soil & 16 & 5 & 0,29 & variable & $8 / 38$ & $-25 / 5$ & 150 & 1.5 \\
\hline Obstacle & - & 0.5 & 0.29 & - & - & - & - & - \\
\hline
\end{tabular}




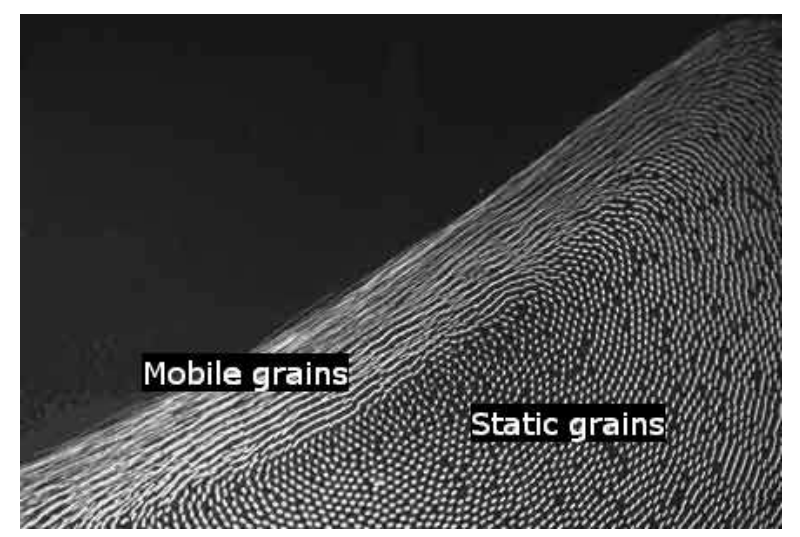

Figure 1: Static and mobile phases of a dry granular media in a rotating drum (website: http://iramis.cea.fr/spec/Pres/Git/GM/gm.htm)

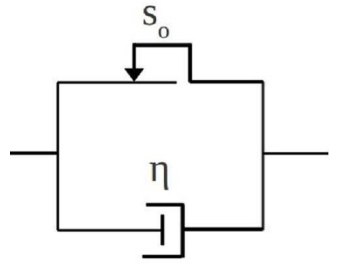

(a)

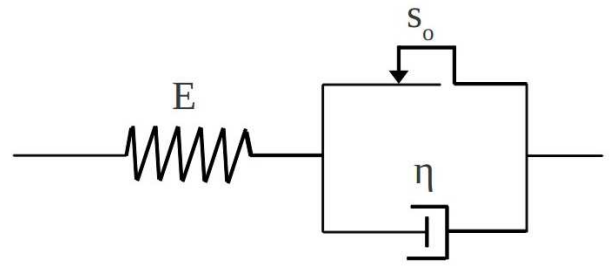

(b)

Figure 2: Bingham (a) and Perzyna (b) models presented in the simplified 1D case

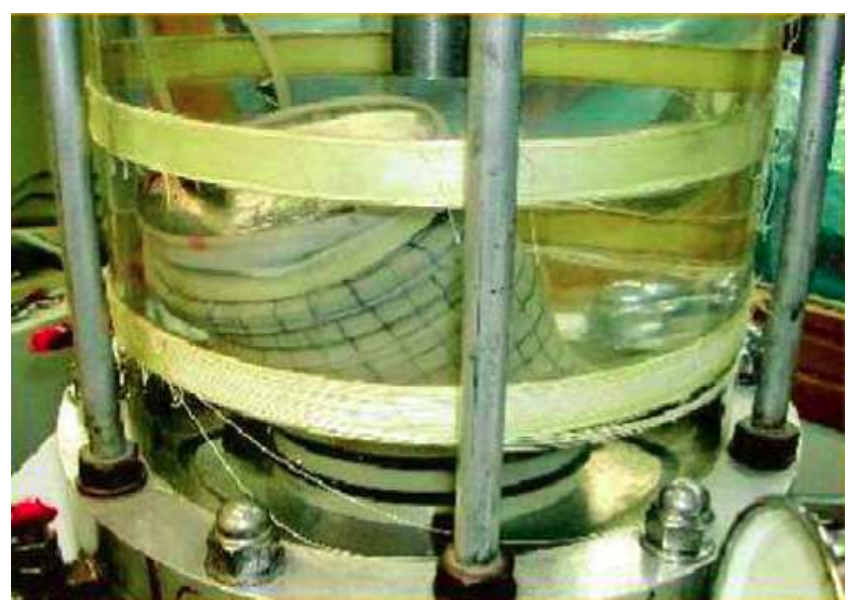

(a)

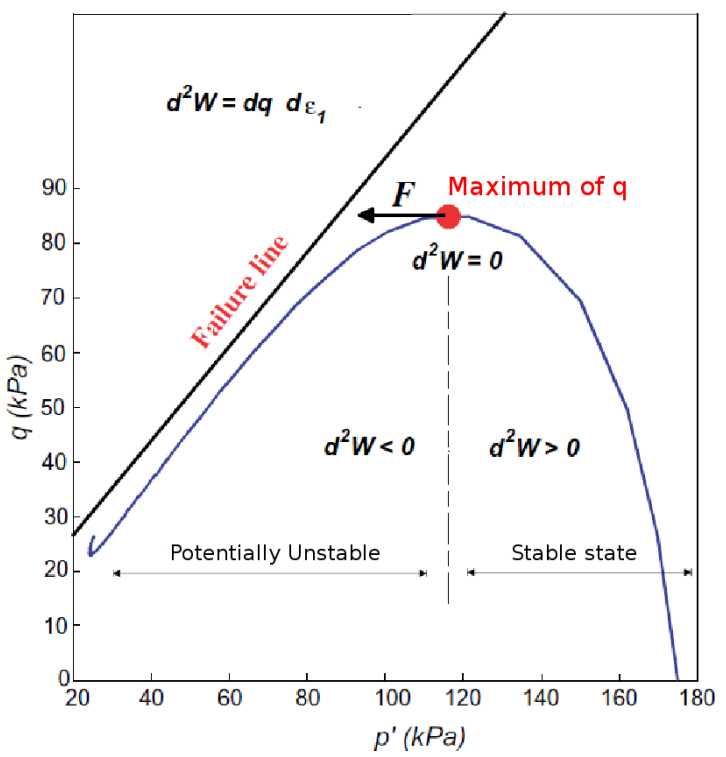

(b)

Figure 3: Loose sand sample after an undrained triaxial [16](a) - Stress path during triaxial test in q-p frame [21] (b) 


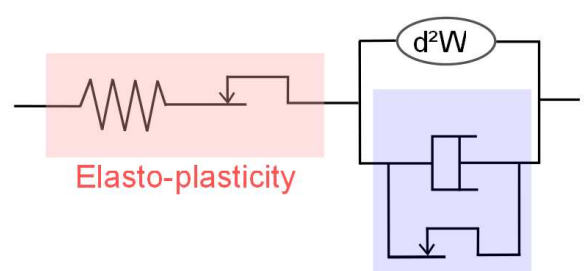

Yield stress viscosity

Figure 4: Scheme of the proposed model in one dimension

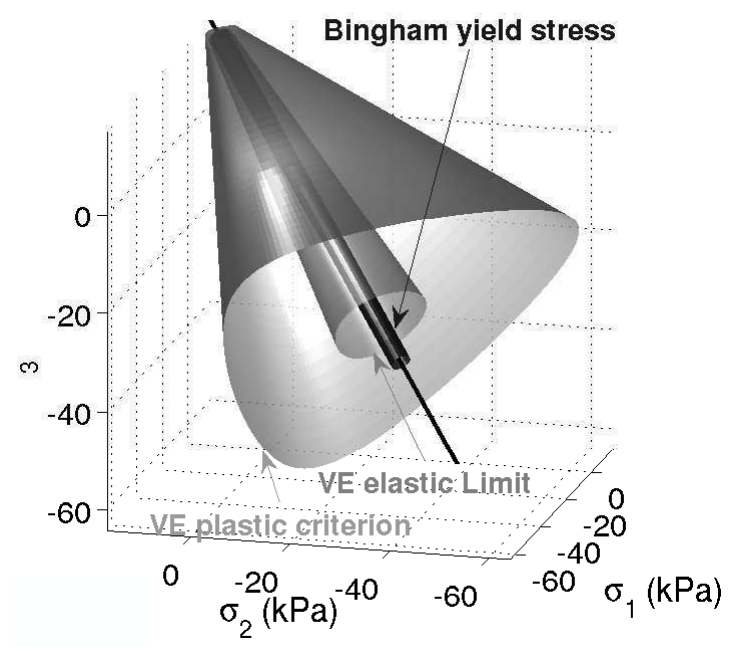

(a) $3 \mathrm{D}$ view

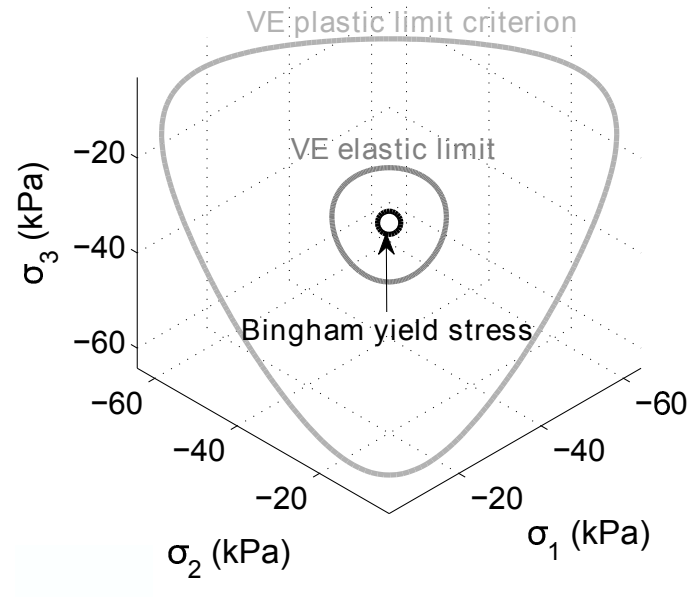

(b) Deviatoric plane view

Figure 5: Characteristic surfaces of the transition model, in the principal stress frame: elastic limit surface, plastic limit criterion, and viscous yield stress ('VE' stands for Van Eekelen)
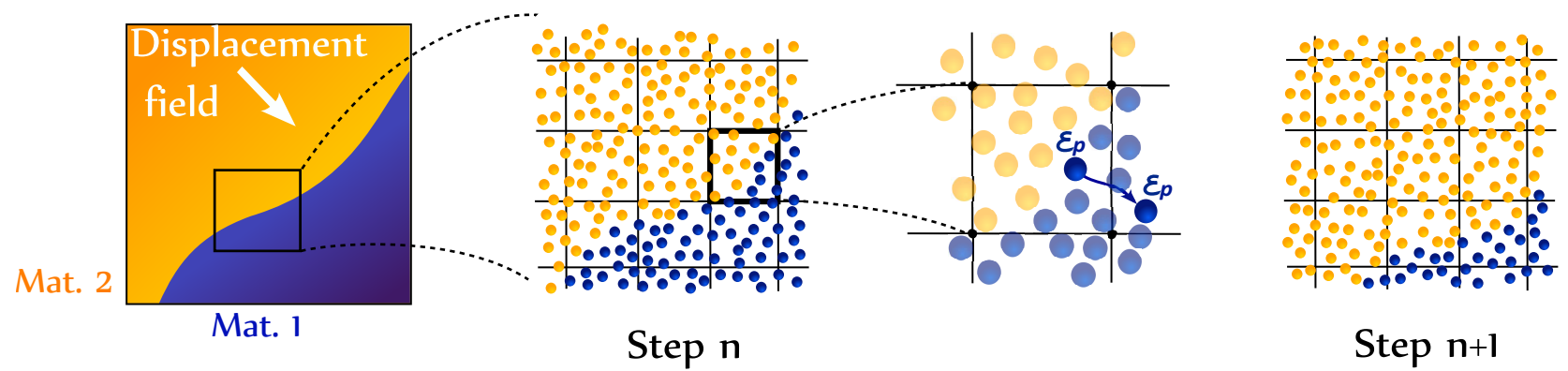

Figure 6: Spatial and material discretizations in FEMLIP. Accurate tracking of the internal variables (as plastic strains) and parameters (represented here by the different colours) during the advection of two materials 


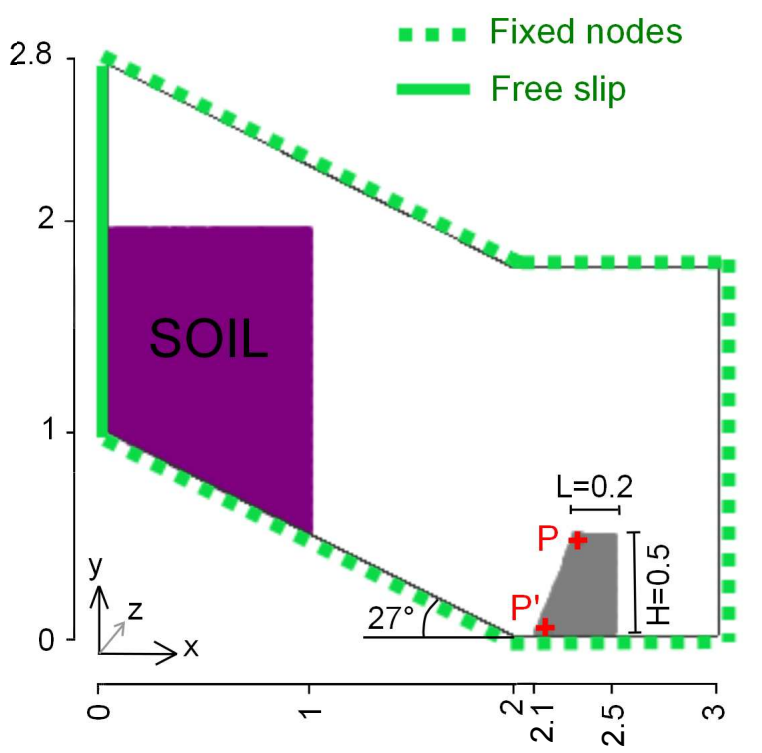

Figure 7: Geometry and boundary conditions of the model studied

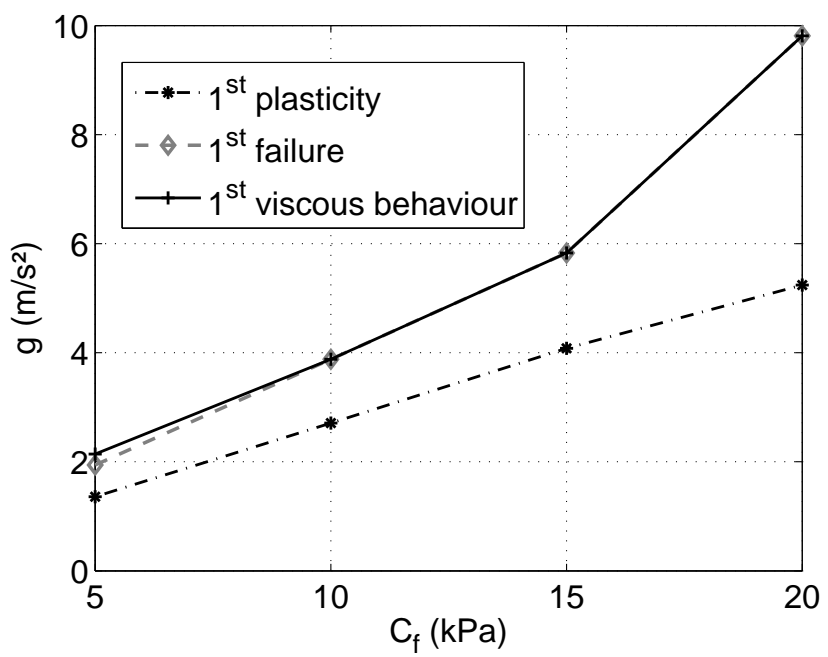

Figure 8: Influence of cohesion on stability

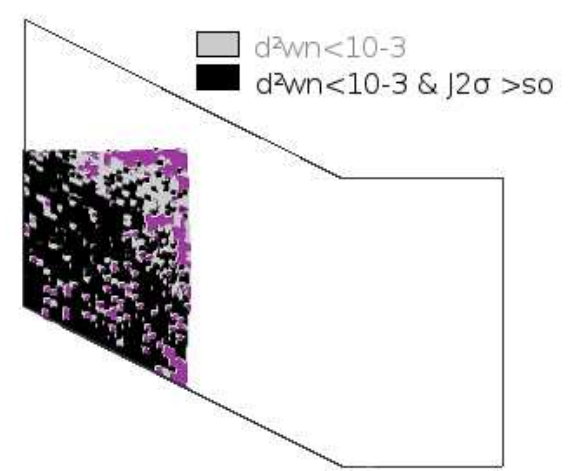

Figure 9: Zoning of failure zones $\left(d^{2} W_{n}<10^{-3}\right)$ and viscous zones $\left(d^{2} W_{n}<10^{-3}\right.$ and $\left.J_{2 \sigma}>s_{o}\right)$ during the loading $\left(g=7.8 \mathrm{~m} / \mathrm{s}^{2}\right)$ and for $C=2-10 \mathrm{kPa}$ 


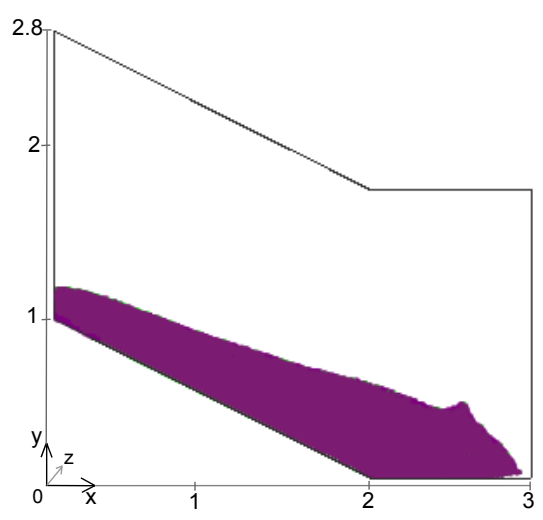

Figure 10: Final configuration without obstacle

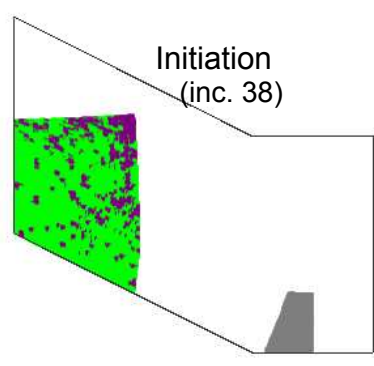

(a)

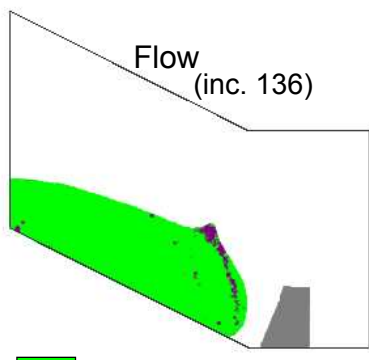

Fluid behaviour

Solid behaviour

(b)

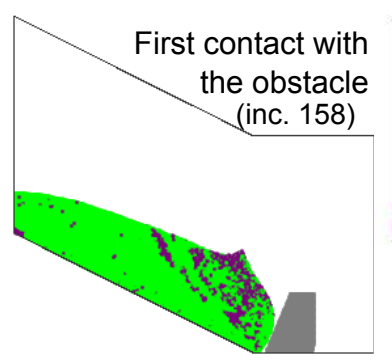

(c)

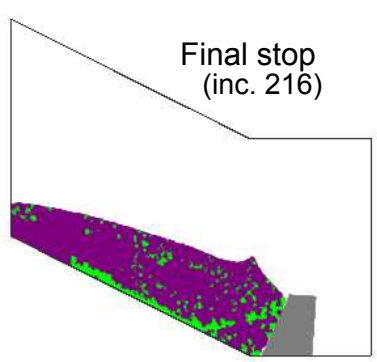

(d)

Figure 11: Different stages of the ash-flow: (a) diffuse failure, (b) general flow, (c) first solidification at the obstacle contact, (d) last remaining viscous band, in the most sheared zone.

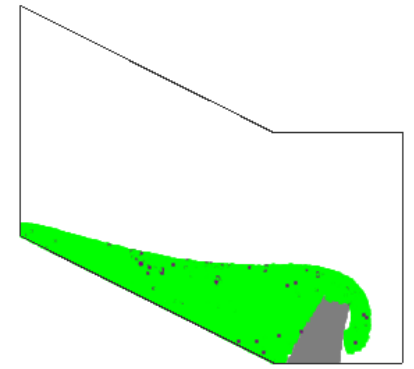

(a) $s_{o}=0.1 \mathrm{kPa}$

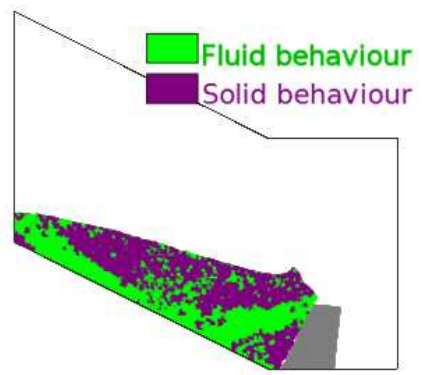

(b) $s_{o}=1.0 \mathrm{kPa}$

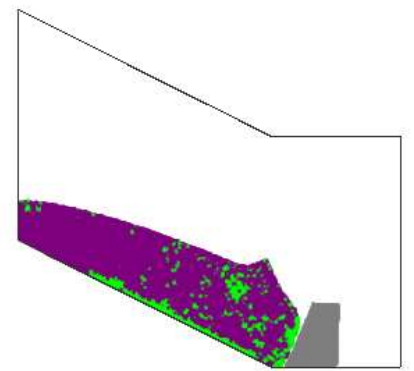

(c) $s_{o}=2.0 \mathrm{kPa}$

Figure 12: Influence of the yield stress viscosity $s_{0}$ on the flow 


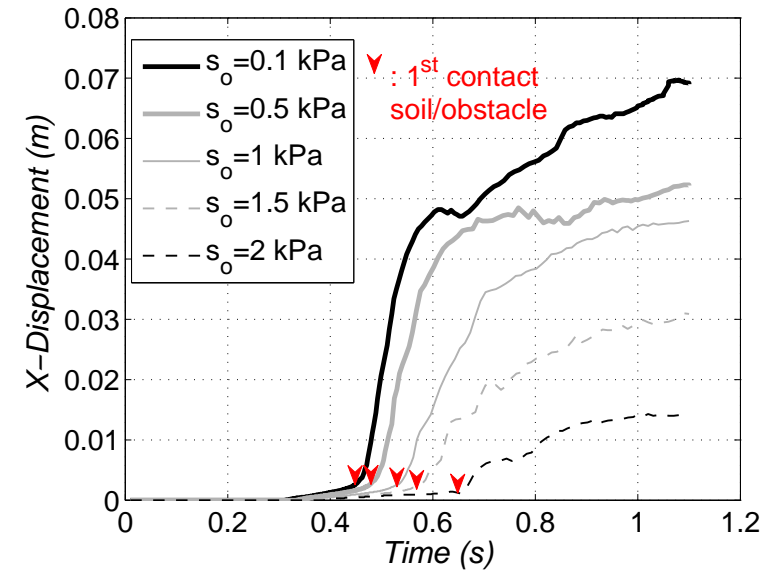

(a) Influence of $s_{o}$ on the horizontal displacement of the obstacle's top

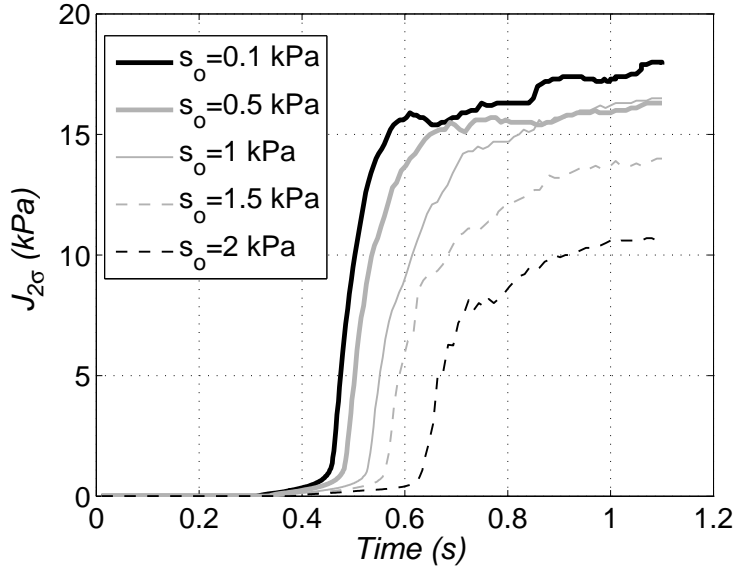

(b) Influence of $s_{o}$ on $J_{2 \sigma}$ of the obstacle's base

Figure 13: Parametric study on the yield stress $s_{o}$

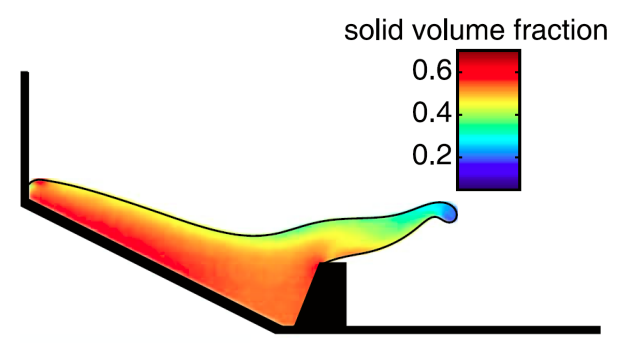

Figure 14: Configuration obtained by [46] at $t=1 \mathrm{~s}$

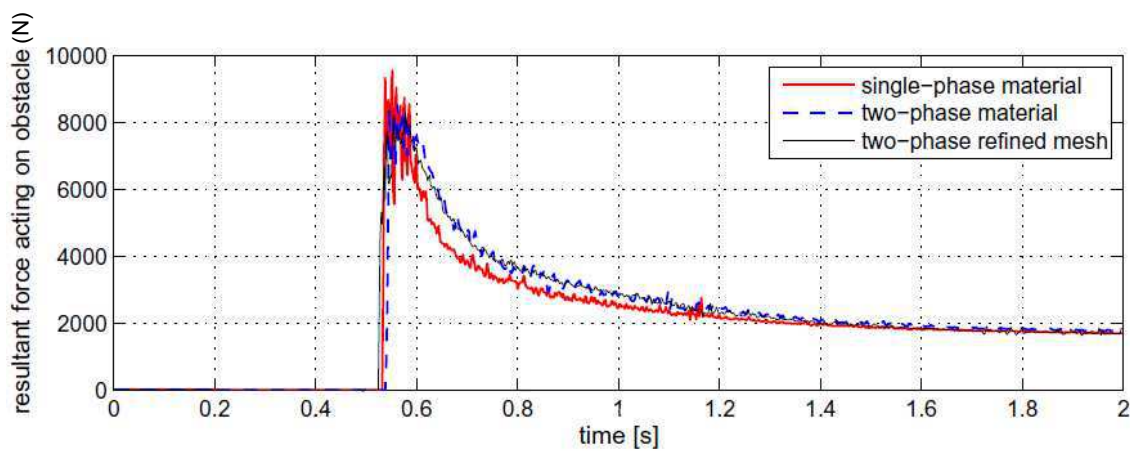

(a) Result obtained by Preisig and Zimmermann [46]

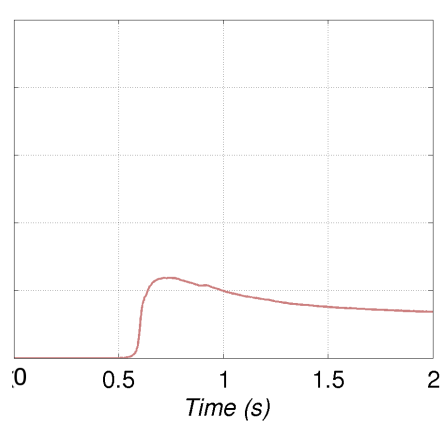

(b) Result with Ellipsis

Figure 15: Comparison of the resulting force obtained with the two simulations 